\title{
Bogdanov-Takens and Triple Zero Bifurcations of a Delayed Modified Leslie-Gower Predator Prey System
}

\author{
Xia Liu and Jinling Wang \\ College of Mathematics and Information Science, Henan Normal University, 453007, China \\ Correspondence should be addressed to Xia Liu; liuxiapost@163.com
}

Received 20 July 2013; Revised 4 September 2013; Accepted 4 September 2013

Academic Editor: Yanni Xiao

Copyright ( 2013 X. Liu and J. Wang. This is an open access article distributed under the Creative Commons Attribution License, which permits unrestricted use, distribution, and reproduction in any medium, provided the original work is properly cited.

A delayed modified Leslie-Gower predator prey system with nonlinear harvesting is considered. The existence conditions that an equilibrium is Bogdanov-Takens (BT) or triple zero singularity of the system are given. By using the center manifold reduction, the normal form theory, and the formulae developed by Xu and Huang, 2008 and Qiao et al., 2010, the normal forms and the versal unfoldings for this singularity are presented. The Hopf bifurcation of the system at another interior equilibrium is analyzed by taking delay (small or large) as bifurcation parameter.

\section{Introduction}

For a more detailed study on the properties of the predator prey systems, the multiple bifurcations for some systems (ODE) with more interior equilibria are investigated by many authors, see [1-4] for example. To deal with this type of systems, the more difficult problem is how to obtain the normal form of the system at its degenerate equilibrium, that is, BT bifurcation.

When introducing the time delay into this type of systems, using the methods developed by [5], the authors in $[6,7]$ have researched the BT bifurcation of some predator prey systems (DDE). Their results show that time delay may have an effect or not on the BT bifurcation.

Recently, papers [8-10] have considered the triple zero bifurcation of some delay differential equations, depending on the parameters in the original system; some interesting bifurcation results are obtained. But we find that there are few results about the triple zero bifurcation for predator prey systems.

Summarizing the above references, we will consider the following predator prey system with Michaelis-Menten type (nonlinear) prey harvesting:

$$
\begin{gathered}
\dot{x}=r x\left(1-\frac{x}{K}\right)-\frac{a_{1} x y}{n+x}-\frac{q E x}{k_{1} E+k_{2} x}, \\
\dot{y}=s_{1} y\left(1-\frac{a_{2} y(t-\tau)}{n+x(t-\tau)}\right),
\end{gathered}
$$

where $x$ and $y$ denote the prey and predator populations, respectively. $\tau$ represents the negative feedback of the predator's density. For complete reason, we give the biological meaning of the parameters, one can be seeing them in [11]. $r$ and $K$ are intrinsic growth rate and environmental carrying capacity for the prey, respectively. $a_{1}$ is the maximum value of the per capita reduction rate of prey, $n$ measures the extent to which the environment provides protection to prey and predator respectively, $s_{1}$ measures the growth rate of the predator species, and $s_{1} a_{2}$ is the maximum value of the per capita reduction rate of predator. $p(x)=q E x /\left(k_{1} E+k_{2} x\right)$ represents Michaelis-Menten type harvesting, $q$ is the catchability coefficient, $E$ is the effort applied to harvest the prey species, and $k_{1}$ and $k_{2}$ are suitable constants.

The authors in $[12,13]$ have studied system (1) without prey harvesting $p(x)$, respectively, in [12], the global stability and persistence of the system are investigated. In [13], by using the Hopf bifurcation theorem and taking the delay as a parameter of bifurcation for small and large cases, the existence of the bifurcated limit cycle around a boundary equilibrium or an interior equilibrium is mainly considered.

For system (1) with $\tau=0$, the authors in [11] have given detailed analysis about the existence of the multiple bifurcations (including BT bifurcation) depending on the parameters of the system. 
For computation simplicity, we first rescale system (1).

Let $\bar{t}=r t, \bar{x}(\bar{t})=x(t) / K, \bar{y}(\bar{t})=a_{1} y(t) / K$, and $\bar{\tau}=r \tau$; then dropping the bars we obtain

$$
\begin{gathered}
\dot{x}=x\left(1-x-\frac{\alpha y}{m+x}-\frac{h}{c+x}\right), \\
\dot{y}=\rho y\left(1-\frac{\beta y(t-\tau)}{m+x(t-\tau)}\right),
\end{gathered}
$$

where $\alpha=1 / r, \beta=a_{2} / a_{1}, m=n / K, \rho=s_{1} / r, h=q E / r k_{2} K$, and $c=k_{1} E / k_{2} K$.

System (2) with initial conditions is

$$
\begin{array}{ll}
\tilde{\phi}(\vartheta) \geqslant 0, & \widetilde{\psi}(\vartheta) \geqslant 0, \\
\vartheta \in[-\tau, 0), & \widetilde{\phi}(0)>0, \quad \widetilde{\psi}(0)>0,
\end{array}
$$

where $\widetilde{\phi}(\vartheta)$ and $\widetilde{\psi}(\vartheta)$ are all continuous bounded functions in the interval $[-\tau, 0)$.

From [11], we know that (2) has interior equilibrium $\bar{E}=$ $(\bar{x}, \bar{y})$ if

$$
h=h^{*}=\frac{1}{4}\left(1-\frac{\alpha}{\beta}+c\right)^{2}=(c+\bar{x})^{2}, \quad \frac{\alpha}{\beta}+c<1,
$$

where $\bar{x}=(1 / 2)(1-c-\alpha / \beta), \bar{y}=(m+\bar{x}) / \beta$ and interior equilibrium $E^{*}=\left(x^{*}, y^{*}\right)$ if $h<h^{*}$, where $x^{*}=(1 / 2)(1-$ $c-\alpha / \beta+\sqrt{\Delta}), y^{*}=\left(m+x^{*}\right) / \beta$, and $\Delta=(\alpha / \beta+c-1)^{2}-$ $4 c(\alpha / \beta+h / c-1)$.

In this paper, for system (2), we will mainly consider the BT and triple zero bifurcations at $\bar{E}$ and the Hopf bifurcation at $E^{*}$. It is easy to see that this system is with six parameters which will let our work become more challenging. When dealing with the BT and triple bifurcations of the delay systems, the core problem is to change delay systems as ordinary differential systems (ODEs).

The concrete organization of the paper is as follows: in Section 2, we will give the conditions under which the equilibrium $\bar{E}$ is a BT singularity, and a universal unfolding will be exhibited; in Section 3, when $\bar{E}$ is a triple zero, the universal unfolding will be presented, and in Section 4 , some Hopf bifurcation results at $E^{*}$ will be obtained.

\section{Bogdanov-Takens Bifurcation}

System (2) also can be written as

$$
\begin{gathered}
\dot{x}=\tau x\left(1-x-\frac{\alpha y}{m+x}-\frac{h}{c+x}\right), \\
\dot{y}=\tau \rho y\left(1-\frac{\beta y(t-1)}{m+x(t-1)}\right) .
\end{gathered}
$$

Linearizing system (5) at $\bar{E}$ yields the following linear system

$$
\begin{gathered}
\dot{x}_{1}=\frac{\tau \alpha \bar{x}}{\beta(m+\bar{x})} x_{1}(t)-\frac{\tau \alpha \bar{x}}{m+\bar{x}} x_{2}(t), \\
\dot{x}_{2}=\frac{\tau \rho}{\beta} x_{1}(t-1)-\tau \rho x_{2}(t-1),
\end{gathered}
$$

the corresponding characteristic equation is

$$
F(\lambda)=\lambda^{2}+\left(\tau \rho e^{-\lambda}-\frac{\tau \alpha \bar{x}}{\beta(m+\bar{x})}\right) \lambda .
$$

Evidently, $\lambda=0$ is a double zero eigenvalue if

$$
\tau \neq \frac{1}{\rho}, \quad \rho=\frac{\alpha \bar{x}}{\beta(m+\bar{x})} ;
$$

$\lambda=0$ is a triple zero eigenvalue if

$$
\tau=\frac{1}{\rho}, \quad \rho=\frac{\alpha \bar{x}}{\beta(m+\bar{x})} .
$$

It is easy to prove that in the above two cases the rest eigenvalues all have negative real parts.

Under the conditions (4) and (8), let $x_{1}=x-\bar{x}$, and let $x_{2}=y-\bar{y}$; then the Taylor expansion system (5) at $\bar{E}$ is

$$
\begin{aligned}
\dot{x}_{1}= & \tau \rho x_{1}(t)-\tau \beta \rho x_{2}(t)+\sum_{i+j \geq 2} \frac{1}{i ! j !} f_{i j}^{(1)} x_{1}^{i}(t) x_{2}^{j}(t), \\
\dot{x}_{2}= & \frac{\tau \rho}{\beta} x_{1}(t-1)-\tau \rho x_{2}(t-1) \\
& +\sum_{i+j+k \geq 2} \frac{1}{i ! j ! k !} f_{i j k}^{(2)} x_{1}^{i}(t-1) x_{2}^{j}(t-1) x_{2}^{k}(t),
\end{aligned}
$$

where $i, j, k \geq 0, f_{i j}^{(1)}=\left.\left(\partial^{i+j} f^{(1)} / \partial^{i} x \partial^{j} y\right)\right|_{(\bar{x}, \bar{y})}, f_{i j k}^{(2)}=$ $\left.\left(\partial^{i+j+k} f^{(2)} / \partial^{i} x \partial^{j} y \partial^{k} y_{0}\right)\right|_{(\bar{x}, \bar{y})}, f^{(1)}=\tau x(1-x-\alpha y /(m+x)-$ $h /(c+x)), f^{(2)}=\tau y_{0}(1-\beta y /(m+x))$.

In the following, we first give the normal form of the system $(10)$ at the singularity $(0,0)$. Reference [6], we first rewrite system $(10)$ as $\dot{X}(t)=L\left(X_{t}\right)$, here $X(t)=\left(x_{1}(t), x_{2}(t)\right)$, $L(\phi)=L\left(\begin{array}{c}\phi_{1}(-1) \\ \phi_{2}(0)\end{array}\right)$, and $\phi=\left(\phi_{1}, \phi_{2}\right)$. By the normal form theory developed by Faria and Magalhaes [5], one can obtain the center manifold of this system at the origin which is twodimensional and system can be reduced to an ODE in the plane.

Define $A_{0}$ to be the infinitesimal generator of system. Consider $\lambda=\{0\}$ and let $P$ denote the invariant space of $A_{0}$ associated with the eigenvalue $\lambda=0$, using the formal adjoint theory in [5], the phase space $C_{1}$ can be decomposed by $\lambda$ as $C_{1}=P \oplus Q$. Let $\Phi$ and $\Psi$ be the bases for $P$ and $P^{*}$, respectively, and be let them normalized such that $\langle\Psi, \Phi\rangle=I$, $\dot{\Phi}=\Phi J$, and $\dot{\Psi}=-J \Psi$, where $\Phi$ and $\Psi$ are $2 \times 2$ matrices, where $J=\left(\begin{array}{ll}0 & 1 \\ 0 & 0\end{array}\right)$.

Next, we will find the $\Phi(\theta)$ and $\Psi(s)$ based on the techniques developed by [14].

Lemma 1 (see Xu and Huang [14]). The bases of $P$ and their dual space $P^{*}$ have the following representations:

$$
\begin{array}{cl}
P=\operatorname{span} \Phi, & \Phi(\theta)=\left(\varphi_{1}(\theta), \varphi_{2}(\theta)\right), \quad-1 \leq \theta \leq 0, \\
P^{*}=\operatorname{span} \Psi, & \Psi(s)=\operatorname{col}\left(\psi_{1}(s), \psi_{2}(s)\right), \quad 0 \leq s \leq 1,
\end{array}
$$


where $\varphi_{1}(\theta)=\varphi_{1}^{0} \in R^{n} \backslash\{0\}, \varphi_{2}(\theta)=\varphi_{2}^{0}+\varphi_{1}^{0} \theta, \varphi_{2}^{0} \in R^{n}$, and $\psi_{2}(s)=\psi_{2}^{0} \in R^{n *} \backslash\{0\}, \psi_{1}(s)=\psi_{1}^{0}-s \psi_{2}^{0}, \psi_{1}^{0} \in R^{n *}$, which satisfy

(1) $(A+B) \varphi_{1}^{0}=0$,

(2) $(A+B) \varphi_{2}^{0}=(B+I) \varphi_{1}^{0}$,

(3) $\psi_{2}^{0}(A+B)=0$,

(4) $\psi_{1}^{0}(A+B)=\psi_{2}^{0}(B+I)$,

(5) $\psi_{2}^{0} \varphi_{2}^{0}-(1 / 2) \psi_{2}^{0} B \varphi_{1}^{0}+\psi_{2}^{0} B \varphi_{2}^{0}=1$,

(6) $\psi_{1}^{0} \varphi_{2}^{0}-(1 / 2) \psi_{1}^{0} B \varphi_{1}^{0}+\psi_{1}^{0} B \varphi_{2}^{0}+(1 / 6) \psi_{2}^{0} B \varphi_{1}^{0}-$ $(1 / 2) \psi_{2}^{0} B \varphi_{2}^{0}=0$.

By system (6), we know that

$$
\begin{gathered}
A=\left(\begin{array}{cc}
\frac{\tau \alpha \bar{x}}{\beta(m+\bar{x})} & -\frac{\tau \alpha \bar{x}}{m+\bar{x}} \\
0 & 0
\end{array}\right)=\left(\begin{array}{cc}
\tau \rho & -\tau \beta \rho \\
0 & 0
\end{array}\right), \\
B=\left(\begin{array}{cc}
0 & 0 \\
\frac{\tau \rho}{\beta} & -\tau \rho
\end{array}\right) .
\end{gathered}
$$

By Lemma 1, we have

$$
\begin{gathered}
\Phi(\theta)=\left(\begin{array}{cc}
\beta \frac{\beta\left[2-(\tau \rho-2)^{2}\right]}{2 \tau \rho(\tau \rho-1)}+\beta \theta \\
1 & \frac{2-\tau \rho}{2(\tau \rho-1)}+\theta
\end{array}\right) \\
\triangleq\left(\begin{array}{cc}
\beta & m_{1}+\beta \theta \\
1 & m_{2}+\theta
\end{array}\right), \quad-1 \leq \theta \leq 0, \\
\Psi(s)=\left(\begin{array}{cc}
-\frac{\tau \rho}{\beta(1-\tau \rho)} s & 1-\frac{\tau \rho}{\tau \rho-1} s \\
\frac{\tau \rho}{\beta(1-\tau \rho)} & \frac{\tau \rho}{\tau \rho-1}
\end{array}\right), \quad 0 \leq s \leq 1,
\end{gathered}
$$

where $m_{1}=\beta\left[2-(\tau \rho-2)^{2}\right] / 2 \tau \rho(\tau \rho-1), m_{2}=(2-\tau \rho) / 2(\tau \rho-$ $1)$,

$$
\Phi(\theta) z=\left(\begin{array}{cc}
\beta & m_{1}+\beta \theta \\
1 & m_{2}+\theta
\end{array}\right)\left(\begin{array}{c}
z_{1} \\
z_{2}
\end{array}\right)=\left(\begin{array}{c}
\beta z_{1}+\left(m_{1}+\beta \theta\right) z_{2} \\
z_{1}+\left(m_{2}+\theta\right) z_{2}
\end{array}\right),
$$

therefore,

$$
\begin{gathered}
\phi_{1}(0)=\beta z_{1}+m_{1} z_{2}, \quad \phi_{2}(0)=z_{1}+m_{2} z_{2}, \\
\phi_{1}(-1)=\beta z_{1}+\left(m_{1}-\beta\right) z_{2}, \\
\phi_{2}(-1)=z_{1}+\left(m_{2}-1\right) z_{2} .
\end{gathered}
$$

Taking a similar theory in [6], system (6) in the center manifold is

$$
\dot{z}=J z+\Psi(0) F(\Phi z)
$$

where

$$
F(\phi)=\left(\begin{array}{c}
\tau \sum_{i+j \geq 2} \frac{1}{i ! j !} f_{i j}^{(1)}\left[\phi_{1}(0)\right]^{i}\left[\phi_{2}(0)\right]^{j} \\
\tau \rho \sum_{i+j+k \geq 2} \frac{1}{i ! j ! k !} f_{i j k}^{(2)} \phi_{1}^{i}(-1) \phi_{2}^{j}(-1) \phi_{2}^{k}(0)
\end{array}\right) .
$$

Using (10) and (15), system (16) also can be written as

$$
\begin{gathered}
\dot{z}_{1}=z_{2}+\frac{\beta(\tau \rho-1)}{\tau \rho(m+\bar{x})} z_{2}^{2}+\text { h.o.t, } \\
\dot{z}_{2}=g_{1} z_{1}^{2}+g_{2} z_{1} z_{2}+g_{3} z_{2}^{2}+\text { h.o.t, }
\end{gathered}
$$

where $g_{1}=-\tau^{2} \beta \rho \bar{x} /(1-\tau \rho)(c+\bar{x}), g_{2}=\tau \alpha m /(1-$ $\tau \rho)(m+\bar{x})^{2}-2 m_{1} \rho \tau^{2} \bar{x} /(1-\tau \rho)(c+\bar{x}), g_{3}=(\tau \rho / \beta(1-$ $\tau \rho))\left[\alpha m_{1} m / \rho(m+\bar{x})^{2}-\tau \bar{x} m_{1}^{2} /(c+\bar{x})\right]+\beta /(m+\bar{x})$. Through a series of transformation, system (18) becomes

$$
\begin{gathered}
\dot{z}_{1}=z_{2}+\text { h.o.t, } \\
\dot{z}_{2}=d_{1} z_{1}^{2}+d_{2} z_{2}^{2}+\text { h.o.t, }
\end{gathered}
$$

where $d_{1}=g_{1}$ and $d_{2}=g_{2}$. If $d_{1} d_{2} \neq 0$, we have the following theorem.

Theorem 2. Let (4), (8), and $\tau \neq \beta m(c+\bar{x}) / 2 m_{1} \bar{x}^{2}(m+\bar{x})$ hold. Then, the equilibrium $\bar{E}$ of system (5) is a BT singularity.

Next, we are interested in giving a versal unfolding for system (5) at BT singularity. Choosing $h$ and $\rho$ as bifurcation parameters and incorporating $h+\lambda_{1}$ and $\rho+\lambda_{2}$ to system (5), where $\lambda_{1}$ and $\lambda_{2}$ vary in a small neighborhood of $(0,0)$, we obtain

$$
\begin{gathered}
\dot{x}=\tau x\left(1-x-\frac{\alpha y}{m+x}-\frac{h+\lambda_{1}}{c+x}\right), \\
\dot{y}=\tau\left(\rho+\lambda_{2}\right) y\left(1-\frac{\beta y(t-1)}{m+x(t-1)}\right) .
\end{gathered}
$$

Let $y_{1}=x-\bar{x}$ and $y_{2}=y-\bar{y}$. Then, system (20) becomes

$$
\begin{aligned}
\dot{y}_{1}= & -\frac{\tau \bar{x} \lambda_{1}}{c+\bar{x}}+\tau\left[\frac{\alpha \bar{x}}{\beta(m+\bar{x})}+\frac{\bar{x} \lambda_{1}}{h}-\frac{\lambda_{1}}{c+\bar{x}}\right] y_{1}(t) \\
& -\frac{\tau \alpha \bar{x}}{m+\bar{x}} y_{2}(t)+\left[\frac{\tau \alpha m}{\beta(m+\bar{x})^{2}}+\frac{\tau\left(c \lambda_{1}-h \bar{x}\right)}{h(c+\bar{x})}\right] y_{1}^{2}(t) \\
& -\frac{\tau \alpha m}{(m+\bar{x})^{2}} y_{1}(t) y_{2}(t)+\text { h.o.t, } \\
\dot{y}_{2}= & \tau\left(\rho^{*}+\lambda_{2}\right) \\
& \times\left[\frac{1}{\beta} y_{1}(t-1)-y_{2}(t-1)+\frac{1}{m+\bar{x}} y_{1}(t-1) y_{2}(t)\right. \\
& \quad+\frac{1}{m+\bar{x}} y_{1}(t-1) y_{2}(t-1)-\frac{1}{\beta(m+\bar{x})} y_{1}^{2}(t-1) \\
& \left.\quad-\frac{\beta}{m+\bar{x}} y_{2}(t) y_{2}(t-1)\right]+ \text { h.o.t. }
\end{aligned}
$$


Then, system (21) can be decomposed as

$$
\begin{aligned}
\dot{z}= & B_{1}+B_{2} z+\Psi_{0} G(\Phi z+u), \\
\dot{u}= & A_{Q^{\prime}} u+(I-\pi) X_{0} \\
& \quad \times\left[B_{0}+B_{2}\left(\Phi_{0} z+u(0)\right)+G(\Phi z+u)\right],
\end{aligned}
$$

where

$$
\begin{gathered}
X_{0}(\theta)= \begin{cases}I, & \theta=0 \\
0, & -1 \leq \theta<0,\end{cases} \\
B_{0}=\left(\begin{array}{c}
-\frac{\tau \bar{x} \lambda_{1}}{c+\bar{x}} \\
0
\end{array}\right), \\
B_{1}=\Psi(0) B_{0}=\left(\begin{array}{cc}
\frac{\tau \rho}{\beta(1-\tau \rho)} & \frac{\tau \rho}{\tau \rho-1}
\end{array}\right)\left(\begin{array}{c}
-\frac{\tau \bar{x} \lambda_{1}}{c+\bar{x}} \\
0
\end{array}\right) \\
=\left(\begin{array}{c}
0 \\
-\frac{\tau^{2} \rho \bar{x} \lambda_{1}}{\beta(c+\bar{x})(1-\tau \rho)}
\end{array}\right),
\end{gathered}
$$

$G(\phi)$

$$
\begin{aligned}
& =\left(\begin{array}{c}
\tau \sum_{i+j \geq 2} \frac{1}{i ! j !} g_{i j}^{(1)}\left[\phi_{1}(0)\right]^{i}\left[\phi_{2}(0)\right]^{j} \\
\tau\left(\rho+\lambda_{2}\right) \sum_{i+j+k \geq 2} \frac{1}{i ! j ! k !} g_{i j k}^{(2)} \phi_{1}^{i}(-1) \phi_{2}^{j}(-1) \phi_{2}^{k}(0)
\end{array}\right), \\
& g_{i j}^{(1)}=\left.\frac{\partial^{i+j} g^{(1)}}{\partial^{i} x \partial^{j} y}\right|_{\left(\bar{x}, \bar{y}, \lambda_{1}\right)}, \quad g_{i j k}^{(2)}=\left.\frac{\partial^{i+j+k} f^{(2)}}{\partial^{i} x \partial^{j} y \partial^{k} y_{0}}\right|_{(\bar{x}, \bar{y})}, \\
& g^{(1)}=x\left(1-x-\frac{\alpha y}{m+x}-\frac{h^{*}+\lambda_{1}}{c+x}\right), \\
& g^{(2)}=y_{0}\left(1-\frac{\beta y}{m+x}\right) \text {, } \\
& B_{2}=\left(\begin{array}{cc}
0 & 1+\frac{\lambda_{2}}{\rho} \\
\frac{\tau^{2} \rho c \lambda_{1}}{h(\tau \rho-1)} & \frac{\tau^{2} \rho}{\tau \rho-1}\left(\frac{c m_{1} \lambda_{1}}{h \beta}+\frac{\lambda_{2}}{\tau \rho}\right)
\end{array}\right) .
\end{aligned}
$$

Then, the normal form of system (21) at $\bar{E}$ is $\dot{z}=B_{1}+B_{2} z+$ $\Psi(0) G(\Phi z)$; that is,

$$
\begin{aligned}
\dot{z}_{1}=z_{2}+ & \frac{\lambda_{2}}{\rho} z_{2}+\frac{\beta\left(\rho+\lambda_{2}\right)(\tau \rho-1)}{\tau \rho^{2}(m+\bar{x})} z_{2}^{2}+\text { h.o.t, } \\
\dot{z}_{2}= & -\frac{\tau^{2} \bar{x} \rho \lambda_{1}}{\beta(c+\bar{x})(1-\tau \rho)}+\frac{\tau^{2} c \rho \lambda_{1}}{h(\tau \rho-1)} z_{1} \\
& +\frac{\tau^{2} \rho}{\tau \rho-1}\left(\frac{c m_{1} \lambda_{1}}{h \beta}+\frac{\lambda_{2}}{\tau \rho}\right) z_{2}+P_{1} z_{1}^{2} \\
& +P_{2} z_{1} z_{2}+P_{3} z_{2}^{2}+\text { h.o.t, }
\end{aligned}
$$

where

$$
\begin{gathered}
P_{1}=\frac{\tau^{2} \beta \rho\left(c \lambda_{1}-h \bar{x}\right)}{h(1-\tau \rho)(c+\bar{x})}, \\
P_{2}=\frac{\tau \alpha m}{(1-\tau \rho)(m+\bar{x})^{2}}+\frac{2 m_{1} \rho \tau^{2}\left(c \lambda_{1}-h \bar{x}\right)}{h(1-\tau \rho)(c+\bar{x})}, \\
P_{3}=\frac{\tau \rho}{\beta(1-\tau \rho)}\left[\frac{\alpha m_{1} m}{\rho(m+\bar{x})^{2}}+\frac{\tau\left(c \lambda_{1}-h \bar{x}\right) m_{1}^{2}}{h(c+\bar{x})}\right] \\
+\frac{\beta\left(\rho+\lambda_{2}\right)}{\rho(m+\bar{x})} .
\end{gathered}
$$

Following the normal form formula in Kuznetsov [15], system (24) can be reduced to

$$
\begin{gathered}
\dot{z}_{1}=z_{2}+\text { h.o.t } \\
\dot{z}_{2}=\gamma_{1}+\gamma_{2} z_{1}+d_{1} z_{1}^{2}+d_{2} z_{1} z_{2}+\text { h.o.t }
\end{gathered}
$$

where

$$
\begin{gathered}
\gamma_{1}=-\frac{\tau^{2} \bar{x} \rho \lambda_{1}}{\beta(c+\bar{x})(1-\tau \rho)}, \\
\gamma_{2}=\left[\frac{2 \tau^{2} \bar{x}}{c+\bar{x}}\left(\frac{2 \beta^{2} \bar{x}^{2}-\tau c m_{1} \alpha \bar{x}^{2}(c+\bar{x})}{\rho m \beta^{2}(c+\bar{x})-2 m_{1} \tau \alpha \bar{x}^{3}}\right)\right. \\
\left.+\frac{\rho^{3} \tau^{3}\left(m_{1} m \beta^{2}-\tau m_{1}^{2} \alpha \bar{x}^{3}(c+\bar{x})\right)}{\alpha \bar{x} \beta^{2}(1-\tau \rho)^{2}(c+\bar{x})}\right] \lambda_{1} \\
-\frac{2 \alpha \tau^{2} \bar{x}^{3}}{\rho(1-\tau \rho)\left[m \beta(c+\bar{x})-2 m_{1} \tau \bar{x}^{2}(m+\bar{x})\right]} \lambda_{2} .
\end{gathered}
$$

Then, system (5) exists in the following bifurcation curves in a small neighborhood of the origin in the $\left(\lambda_{1}, \lambda_{2}\right)$ plane.

Theorem 3. Let (4), (8), and $\tau \neq \beta m(c+\bar{x}) / 2 m_{1} \bar{x}^{2}(m+\bar{x})$ hold. System (5) admits the following bifurcations:

(i) a saddle-node bifurcation curve $S N=\left\{\left(\lambda_{1}, \lambda_{2}\right) ; \gamma_{1}=\right.$ $\left.\left(1 / 4 d_{1}\right) \gamma_{2}^{2}\right\}$

(ii) a Hopf bifurcation curve $H=\left\{\left(\lambda_{1}, \lambda_{2}\right) ; \lambda_{2}=0, \gamma_{2}<\right.$ $0\}$;

(iii) a homoclinic bifurcation curve $H L=\left\{\left(\lambda_{1}, \lambda_{2}\right) ; \gamma_{1}=\right.$ $\left.-\left(6 / 25 d_{1}\right) \gamma_{2}^{2}, \gamma_{2}<0\right\}$

\section{Triple-Zero Bifurcation}

From Section 2, we know that under the conditions (4) and (9) the equilibrium $\bar{E}$ of system (5) is a triple zero singularity. In the following reference, from the work of $[8,9]$ we will give the triple zero bifurcation at $\bar{E}$. 
Let $x_{1}=x-\bar{x}$ and $x_{2}=y-\bar{y}$; then, system (5) becomes

$$
\begin{aligned}
\dot{x}_{1}=\tau & {\left[\left(1-2 \bar{x}-\frac{\alpha m}{\beta(m+\bar{x})}-\frac{h c}{(c+\bar{x})^{2}}\right) x_{1}(t)\right.} \\
& \left.-\rho \beta x_{2}(t)+\sum_{i+j \geq 2} \frac{1}{i ! j !} f_{i j}^{(1)} x_{1}^{i}(t) x_{2}^{j}(t)\right], \\
\dot{x}_{2}=\tau \rho & {\left[\frac{1}{\beta} x_{1}(t-1)-x_{2}(t-1)\right.} \\
& \left.+\sum_{i+j+k \geq 2} \frac{1}{i ! j ! k !} f_{i j k}^{(2)} x_{1}^{i}(t-1) x_{2}^{j}(t-1) x_{2}^{k}(t)\right] .
\end{aligned}
$$

To determine a versal unfolding for the original system (28) at $\bar{E}$, we choose $\tau, \rho$, and $h$ as bifurcation parameters, and let them become $\tau+\mu_{1}, \rho+\mu_{2}$, and $h+\mu_{3}$, respectively, where $\mu_{1}, \mu_{2}$, and $\mu_{3}$ vary in a small neighborhood of $(0,0,0)$; then (28) can be written as

$$
\begin{aligned}
& \dot{x}_{1}=(\left.+\mu_{1}\right) \\
& \times[\left(\rho-\frac{c \mu_{3}}{h}\right) x_{1}(t)-\rho \beta x_{2}(t) \\
&+\left(\frac{\alpha m}{\beta(m+\bar{x})^{2}}+\frac{\left(c \mu_{3}-h \bar{x}\right)}{h(c+\bar{x})}\right) x_{1}^{2}(t) \\
&\left.-\frac{\alpha m}{(m+\bar{x})^{2}} x_{1}(t) x_{2}(t)\right]+ \text { h.o.t, } \\
& \dot{x}_{2}=\left(\tau+\mu_{1}\right)\left(\rho+\mu_{2}\right) \\
& \times\left[\frac{1}{\beta} x_{1}(t-1)-x_{2}(t-1)+\frac{1}{m+\bar{x}} x_{1}(t-1) x_{2}(t)\right. \\
& \quad+\frac{1}{m+\bar{x}} x_{1}(t-1) x_{2}(t-1) \\
&\left.\quad-\frac{1}{\beta(m+\bar{x})} x_{1}^{2}(t-1)-\frac{\beta}{m+\bar{x}} x_{2}(t) x_{2}(t-1)\right]
\end{aligned}
$$

+ h.o.t.

Next, we need to find the expressions of $\Phi(\theta)$ and $\Psi(s)$ based on the techniques developed by [9].

Lemma 4 (see Qiao et al. [9]). The bases of $P$ and their dual space $P^{*}$ have the following representations:

$$
\begin{array}{r}
P=\operatorname{span} \Phi, \quad \Phi(\theta)=\left(\varphi_{1}(\theta), \varphi_{2}(\theta), \varphi_{3}(\theta)\right), \\
-1 \leq \theta \leq 0, \\
P^{*}=\operatorname{span} \Psi, \quad \Psi(s)=\operatorname{col}\left(\psi_{1}(s), \psi_{2}(s), \psi_{3}(s)\right), \\
0 \leq s \leq 1,
\end{array}
$$

where $\varphi_{1}(\theta)=\varphi_{1}^{0} \in R^{n} \backslash\{0\}, \varphi_{2}(\theta)=\varphi_{2}^{0}+\varphi_{1}^{0} \theta, \varphi_{3}(\theta)=$ $\varphi_{3}^{0} \varphi_{2}^{0} \theta+(1 / 2) \varphi_{1}^{0} \theta^{2}, \varphi_{2}^{0}, \varphi_{3}^{0} \in R^{n}$, and $\psi_{3}(s)=\psi_{3}^{0} \in R^{n *} \backslash\{0\}$, $\psi_{2}(s)=\psi_{2}^{0}-s \psi_{3}^{0}, \psi_{1}(s)=\psi_{1}^{0}-s \psi_{2}^{0}+(1 / 2) s^{2} \psi_{3}^{0}, \psi_{1}^{0}, \psi_{2}^{0} \in R^{n *}$, which satisfy

(1) $(A+B) \varphi_{1}^{0}=0$,

(2) $(A+B) \varphi_{2}^{0}=(B+I) \varphi_{1}^{0}$,

(3) $(A+B) \varphi_{3}^{0}=(B+I) \varphi_{2}^{0}-(1 / 2) B \varphi_{1}^{0}$,

(4) $\psi_{3}^{0}(A+B)=0$,

(5) $\psi_{2}^{0}(A+B)=\psi_{3}^{0}(B+I)$,

(6) $\psi_{1}^{0}(A+B)=\psi_{2}^{0}(B+I)-(1 / 2) \psi_{3}^{0} B$,

(7) $\psi_{3}^{0}(B+I) \varphi_{3}^{0}-(1 / 2) \psi_{3}^{0} B \varphi_{2}^{0}+(1 / 6) \psi_{3}^{0} B \varphi_{1}^{0}=1$,

(8) $\psi_{2}^{0}(B+I) \varphi_{3}^{0}-(1 / 2) \psi_{2}^{0} B \varphi_{2}^{0}+(1 / 6) \psi_{2}^{0} B \varphi_{1}^{0}-(1 / 2) \psi_{3}^{0} B \varphi_{3}^{0}+$ $(1 / 6) \psi_{3}^{0} B \varphi_{2}^{0}-(1 / 24) \psi_{3}^{0} B \varphi_{1}^{0}=0$,

(9) $\psi_{1}^{0}(B+I) \varphi_{3}^{0}-(1 / 2) \psi_{1}^{0} B \varphi_{2}^{0}+(1 / 6) \psi_{1}^{0} B \varphi_{1}^{0}-$ $(1 / 2) \psi_{2}^{0} B \varphi_{3}^{0}+(1 / 6) \psi_{2}^{0} B \varphi_{2}^{0}-(1 / 24) \psi_{2}^{0} B \varphi_{1}^{0}+$ $(1 / 6) \psi_{3}^{0} B \varphi_{3}^{0}-(1 / 24) \psi_{3}^{0} B \varphi_{2}^{0}+(1 / 120) \psi_{3}^{0} B \varphi_{1}^{0}=0$.

For system (29), one can see that

$$
A+B=\tau \rho\left(\begin{array}{ll}
1 & -\beta \\
\frac{1}{\beta} & -1
\end{array}\right)=\left(\begin{array}{cc}
1 & -\beta \\
1 & -1 \\
\beta &
\end{array}\right) .
$$

By Lemma 4, we can obtain

$$
\begin{gathered}
\Phi(\theta)=\left(\begin{array}{ccc}
\beta & \beta \theta & \beta\left(1+\frac{1}{2} \theta^{2}\right) \\
1 & -1+\theta & 1-\theta+\frac{1}{2} \theta^{2}
\end{array}\right), \quad-1 \leq \theta \leq 0, \\
\Psi(s)=\left(\begin{array}{cc}
-\frac{17}{18 \beta}-\frac{2 s}{3 \beta}+\frac{s^{2}}{\beta} & \frac{35}{18}+\frac{2 s}{3}-s^{2} \\
\frac{2}{3 \beta}-\frac{2 s}{\beta} & -\frac{2}{3}+2 s \\
\frac{2}{\beta} & -2
\end{array}\right), \\
0 \leq s \leq 1,
\end{gathered}
$$

such that $\langle\Psi, \Phi\rangle=I, \dot{\Phi}=\Phi J$, and $\dot{\Psi}=-J \Psi$, where $J$ is given by $J=\left(\begin{array}{lll}0 & 1 & 0 \\ 0 & 0 & 1 \\ 0 & 0 & 0\end{array}\right)$.

Using the similar methods as used in [9], system (29) can be rewritten as

$$
\dot{x}(t)=A(\mu) x(t)+B(\mu) x(t-1)+F(x(t), x(t-1), \mu),
$$

where $\mu=\left(\mu_{1}, \mu_{2}, \mu_{3}\right) \in R^{3}$ is a parameter vector, $x \in R^{2}$. Let

$$
\begin{aligned}
& \widehat{F}(x(t), x(t-1), \mu) \\
& =(A(\lambda)-A(0)) x(t)+(B(\mu)-B(0)) x(t-1) \\
& \quad+F(x(t), x(t-1), \mu),
\end{aligned}
$$


then, we can expand $\widehat{F}(x(t), x(t-1), \mu)=A_{1} \mu_{1} x(t)+$ $A_{2} \mu_{2} x(t)+A_{3} \mu_{3} x(t)+B_{1} \mu_{1} x(t-1)+B_{2} \mu_{2} x(t-1)+$ $B_{3} \mu_{3} x(t-1)+E_{1} x_{1}(t) x(t-1)+E_{2} x_{2}(t) x(t-1)+F_{1} x_{1}(t) x(t)+$ $F_{2} x_{2}(t) x(t)+G_{1} x_{1}(t-1) x(t-1)+G_{2} x_{2}(t-1) x(t-1)+$ h.o.t, where

$$
\begin{aligned}
& A_{1}=\left(\begin{array}{cc}
\rho & -\beta \rho \\
0 & 0
\end{array}\right), \quad A_{2}=\left(\begin{array}{ll}
0 & 0 \\
0 & 0
\end{array}\right), \quad A_{3}=\left(\begin{array}{cc}
-\frac{c \tau}{h} & 0 \\
0 & 0
\end{array}\right), \\
& B_{1}=\left(\begin{array}{cc}
0 & 0 \\
\frac{\rho}{\beta} & -\rho
\end{array}\right), \quad B_{2}=\left(\begin{array}{cc}
0 & 0 \\
\bar{\tau} & -\tau
\end{array}\right), \quad B_{3}=\left(\begin{array}{ll}
0 & 0 \\
0 & 0
\end{array}\right), \\
& E_{1}=\left(\begin{array}{ll}
0 & 0 \\
0 & 0
\end{array}\right), \quad E_{2}=\left(\begin{array}{cc}
0 & 0 \\
\frac{1}{m+\bar{x}} & -\frac{\beta}{m+\bar{x}}
\end{array}\right), \\
& F_{1}=\left(\begin{array}{c}
\frac{\tau \alpha m}{\beta(m+\bar{x})^{2}}-\frac{\tau \bar{x}}{c+\bar{x}}-\frac{\tau \alpha m}{2(m+\bar{x})^{2}} \\
0
\end{array}\right) \text {, } \\
& F_{2}=\left(\begin{array}{cc}
-\frac{\tau \alpha m}{2(m+\bar{x})^{2}} & 0 \\
0 & 0
\end{array}\right) \\
& G_{1}=\left(\begin{array}{cc}
0 & 0 \\
-\frac{1}{\beta(m+\bar{x})} & \frac{1}{2(m+\bar{x})}
\end{array}\right) \text {, } \\
& G_{2}=\left(\begin{array}{cc}
0 & 0 \\
\frac{1}{2(m+\bar{x})} & 0
\end{array}\right) \text {, } \\
& \varphi_{1}^{0}=\left(\begin{array}{c}
\beta \\
1
\end{array}\right), \quad \varphi_{2}^{0}=\left(\begin{array}{c}
0 \\
-1
\end{array}\right), \quad \varphi_{3}^{0}=\left(\begin{array}{c}
\beta \\
1
\end{array}\right), \\
& \psi_{1}^{0}=\left(\begin{array}{ll}
-\frac{17}{18 \beta} & \frac{35}{18}
\end{array}\right) \text {, } \\
& \psi_{2}^{0}=\left(\frac{2}{3 \beta}-\frac{2}{3}\right), \quad \psi_{3}^{0}=\left(\frac{2}{\beta}-2\right) .
\end{aligned}
$$

Following the formula of Theorem 3.1 in [9], the normal form with versal unfolding of system (29) on the center manifold takes the following form:

$$
\begin{gathered}
\dot{z}_{1}=z_{2}, \\
\dot{z}_{2}=z_{3}, \\
\dot{z}_{3}=l_{1} z_{1}+l_{2} z_{2}+l_{3} z_{3}+\eta_{1} z_{1}^{2}+\eta_{2} z_{2}^{2} \\
+\eta_{3} z_{1} z_{2}+\eta_{4} z_{1} z_{3}+\text { h.o.t, }
\end{gathered}
$$

where

$$
\begin{gathered}
l_{1}=-\frac{2 c \tau}{h} \mu_{3}, \quad l_{2}=-4 \tau \mu_{2}-\frac{2 c \tau}{3 h} \mu_{3}, \\
l_{3}=2 \rho \mu_{1}+\frac{4 \tau}{3} \mu_{2}-\frac{19 c \tau}{18 h} \mu_{3},
\end{gathered}
$$

$$
\begin{gathered}
\eta_{1}=-\frac{2 \tau \bar{x} \beta}{c+\bar{x}}, \quad \eta_{2}=\frac{17 \tau \bar{x} \beta}{9(c+\bar{x})}+\frac{2 \tau \alpha m}{3(m+\bar{x})^{2}}, \\
\eta_{3}=-\frac{4 \tau \bar{x} \beta}{3(c+\bar{x})}+\frac{2 \tau \alpha m}{(m+\bar{x})^{2}} \\
\eta_{4}=\frac{2 \tau \alpha m}{3(m+\bar{x})^{2}}-\frac{19 \tau \bar{x} \beta}{9(c+\bar{x})} .
\end{gathered}
$$

Referring [8], we know that if

$$
\left.\left(\frac{\partial\left(l_{1}, l_{2}, l_{3}\right)}{\partial\left(\mu_{1}, \mu_{2}, \mu_{3}\right)}\right)\right|_{\mu=0} \neq 0, \quad \eta_{1} \eta_{2} \eta_{3} \eta_{4} \neq 0,
$$

that is,

$$
\rho \neq \frac{2 \bar{x}^{2}(m+\bar{x})}{3 m(c+\bar{x})}, \quad \rho \neq \frac{19 \bar{x}^{2}(m+\bar{x})}{6 m(c+\bar{x})},
$$

for the unfolding normal form (36) then there exist the following results.

Theorem 5. Let (4), (9), and (39) hold. For the parameters $\mu_{1}, \mu_{2}$, and $\mu_{3}$ are sufficiently small,

(i) system (36) undergoes a transcritical bifurcation at the origin on the curve

$$
T=\left\{\left(\mu_{1}, \mu_{2}, \mu_{3}\right): \mu_{3}=0\right\},
$$

(ii) system (36) undergoes a Hopf bifurcation at the origin on the curve

$$
\begin{aligned}
& H_{1}=\left\{\left(\mu_{1}, \mu_{2}, \mu_{3}\right):\right. \frac{2 \alpha \bar{x}}{\beta(m+\bar{x})} \mu_{1} \\
&= \frac{19 c \tau}{18(c+\bar{x})^{2}} \mu_{3}-\frac{4 \tau}{3} \mu_{2} \\
&+\frac{3 c \mu_{3}}{6(c+\bar{x})^{2} \mu_{2}+c \mu_{3}}, \\
&\left.\mu_{2}>-\frac{c}{6(c+\bar{x})^{2}} \mu_{3}\right\},
\end{aligned}
$$

(iii) system (36) undergoes a Hopf-bifurcation at the nontrivial equilibrium point on the curve

$\mathrm{H}_{2}$

$$
=\left\{\left(\mu_{1}, \mu_{2}, \mu_{3}\right): l_{3}=\left(\frac{\eta_{4}}{\eta_{1}}-\frac{\eta_{1}}{\eta_{3} l_{1}-\eta_{1} l_{2}}\right) l_{1}, \frac{\eta_{1}}{\eta_{3} l_{1}-\eta_{1} l_{2}}>0\right\},
$$

(iv) system (36) undergoes a Bogdanov-Takens bifurcation at the origin on the curve

$$
B=\left\{\left(\mu_{1}, \mu_{2}, \mu_{3}\right): \mu_{3}=0, \mu_{2}=-\frac{c}{6(c+\bar{x})^{2}} \mu_{3}\right\},
$$


(v) system (36) undergoes a zero-Hopf bifurcation at the origin on the curve

$$
\begin{aligned}
& H_{3} \\
& =\left\{\left(\mu_{1}, \mu_{2}, \mu_{3}\right): \mu_{3}=0, \mu_{1}=\frac{2 \tau \beta(m+\bar{x})}{3 \alpha \bar{x}} \mu_{2}, \mu_{2}>0\right\} .
\end{aligned}
$$

\section{Hopf Bifurcation}

The Jacobian matrix of system (2) at $E^{*}$ takes the following form:

$$
\begin{aligned}
& J\left(E^{*}\right) \\
& =\left(\begin{array}{cc}
x^{*}\left(-1+\frac{\alpha y^{*}}{\left(m+x^{*}\right)^{2}}+\frac{h}{\left(c+x^{*}\right)^{2}}\right) & -\frac{\alpha x^{*}}{m+x^{*}} \\
\frac{\rho}{\beta} e^{-\lambda \tau} & -\rho e^{-\lambda \tau}
\end{array}\right),
\end{aligned}
$$

then, the characteristic equation is

$$
\begin{aligned}
F(\lambda)= & \lambda^{2}+\lambda x^{*}\left(1-\frac{\alpha}{\beta\left(m+x^{*}\right)}-\frac{h}{\left(c+x^{*}\right)^{2}}\right) \\
& +\left(\rho \lambda+\rho x^{*}\left(1-\frac{h}{\left(c+x^{*}\right)^{2}}\right)\right) e^{-\lambda \tau} \\
= & \lambda^{2}+\lambda x^{*}\left(\frac{\sqrt{\Delta}}{c+x^{*}}-\frac{\alpha}{\beta\left(m+x^{*}\right)}\right) \\
& +\left(\rho \lambda+\frac{\rho x^{*} \sqrt{\Delta}}{c+x^{*}}\right) e^{-\lambda \tau} .
\end{aligned}
$$

When $\tau=0$, by Routh-Hurwitz criterion, all roots of (46) have negative real part if

$$
\rho>x^{*}\left(\frac{\alpha}{\beta\left(m+x^{*}\right)}-\frac{\sqrt{\Delta}}{c+x^{*}}\right) .
$$

Using the formula in Theorem 2.4 of [13] or Lemma 2.2 of [16], we have the following theorem.

Theorem 6. Let (47) hold. Then, there exist

$$
\begin{gathered}
w_{0}=\frac{\sqrt{2}}{2} \sqrt{\rho^{2}-A^{2}+\sqrt{\left(\rho^{2}-A^{2}\right)^{2}+4 B^{2}}}, \\
\tau_{k}=\frac{1}{w_{0}} \arccos \frac{(B-A \rho) w_{0}^{2}}{B^{2}+\rho^{2} w_{0}^{2}}+\frac{2 k \pi}{w_{0}}, \quad k=0,1, \ldots
\end{gathered}
$$

such that the positive equilibrium $E^{*}$ is asymptotically stable for $0 \leqslant \tau<\tau_{0}$, moreover, system (2) undergoes Hopf bifurcation at $E^{*}$ for $\tau=\tau_{k}$, where $A=\rho+x^{*}\left(\sqrt{\Delta} /\left(c+x^{*}\right)-\alpha / \beta\left(m+x^{*}\right)\right)$ and $B=\rho x^{*} \sqrt{\Delta} /\left(c+x^{*}\right)$.

In the following, using the Hopf bifurcation theorem for a retarded differential system introduced by [17], the Hopf bifurcations at $E^{*}$ for small delay and large delay are presented.

Using the same methods as the ones used in [13], for small delay, let $e^{-\lambda \tau} \simeq 1-\lambda \tau$, together with (46), the following bifurcation results can be obtained.

Theorem 7. Let $0<\tau<\tilde{\tau}=1 / \rho, \rho<\alpha x^{*} / \beta\left(m+x^{*}\right)$, $\tau_{s}=A / B$ and $w_{s}=\sqrt{B /\left(1-\rho \tau_{s}\right)}$. Then, there exists $\epsilon_{s}>0$ such that for each $0 \leqslant \epsilon<\epsilon_{s}$, system (2) near $E^{*}$ has a family or periodic solutions $\gamma_{s}(\epsilon)$ with period $T_{s}=T_{s}(\epsilon)$ for $\tau=\tau(\epsilon)$ such that $\gamma_{s}(0)=E^{*}, T_{s}(0)=2 \pi / w_{s}$, and $\tau(0)=\tau_{s}$.

For large delay, by [13] we have the following results.

Theorem 8. Let (47) hold. Then there exists $\epsilon_{0}>0$ such that for each $0 \leqslant \epsilon<\epsilon_{0}$ system (2) near $E^{*}$ has a family or periodic solutions $\gamma_{l}(\epsilon)$ with period $T_{l}=T_{l}(\epsilon)$ for $\tau=\tau(\epsilon)$ such that $\gamma_{l}(0)=E^{*}, T_{l}(0)=2 \pi / w_{0}$ and $\tau(0)=\tau_{0}$.

Remark. Because the proofs of Theorems 7 and 8 are the same as the proofs of Theorems 3.4 and 3.5 in [13], we omit them here.

\section{Conflict of Interests}

The authors declare that there is no conflict of interests regarding the publication of this paper.

\section{Acknowledgments}

This paper is supported by NSFC (11226142), the Foundation of Henan Educational Committee (2012A110012), the Foundation of Henan Normal University (2011QK04, 2012PL03), and the Scientific Research Foundation for Ph.D. of Henan Normal University (no. 1001).

\section{References}

[1] D. M. Xiao and S. G. Ruan, "Codimension two bifurcations in a predator-prey system with group defense," International Journal of Bifurcation and Chaos in Applied Sciences and Engineering, vol. 11, no. 8, pp. 2123-2131, 2001.

[2] D. Xiao, W. Li, and M. Han, "Dynamics in a ratio-dependent predator-prey model with predator harvesting," Journal of Mathematical Analysis and Applications, vol. 324, no. 1, pp. 1429, 2006.

[3] L. L. Wang, Y. H. Fan, and W. T. Li, "Multiple bifurcations in a predator-prey system with monotonic functional response," Applied Mathematics and Computation, vol. 172, no. 2, pp. 11031120, 2006.

[4] Y. L. Li and D. M. Xiao, "Bifurcations of a predator-prey system of Holling and Leslie types," Chaos, Solitons \& Fractals, vol. 34, no. 2, pp. 606-620, 2007.

[5] T. Faria and L. T. Magalhaes, "Normal forms for retarded functional-differential equations and applications to BogdanovTakens singularity," Journal of Differential Equations, vol. 122, no. 2, pp. 201-224, 1995.

[6] D. M. Xiao and S. G. Ruan, "Multiple bifurcations in a delayed predator-prey system with nonmonotonic functional response," 
Journal of Differential Equations, vol. 176, no. 2, pp. 494-510, 2001.

[7] J. Xia, Z. Liu, R. Yuan, and S. Ruan, "The effects of harvesting and time delay on predator-prey systems with Holling type II functional response," SIAM Journal on Applied Mathematics, vol. 70, no. 4, pp. 1178-1200, 2009.

[8] S. A. Campbell and Y. Yuan, "Zero singularities of codimension two and three in delay differential equations," Nonlinearity, vol. 21, no. 11, pp. 2671-2691, 2008.

[9] Z. Q. Qiao, X. B. Liu, and D. M. Zhu, "Bifurcation in delay differential systems with triple-zero singularity," Chinese Annals of Mathematics A, vol. 31, no. 1, pp. 59-70, 2010.

[10] X. He, C. D. Li, and Y. L. Shu, "Triple-zero bifurcation in van der Pol's oscillator with delayed feedback," Communications in Nonlinear Science and Numerical Simulation, vol. 17, no. 12, pp. 5229-5239, 2012.

[11] R. P. Gupta and P. Chandra, "Bifurcation analysis of modified Leslie-Gower predator-prey model with Michaelis-Menten type prey harvesting," Journal of Mathematical Analysis and Applications, vol. 398, no. 1, pp. 278-295, 2013.

[12] A. F. Nindjin, M. A. Aziz-Alaoui, and M. Cadivel, "Analysis of a predator-prey model with modified Leslie-Gower and Hollingtype II schemes with time delay," Nonlinear Analysis: Real World Applications, vol. 7, no. 5, pp. 1104-1118, 2006.

[13] R. Yafia, F. E. Adnani, and H. T. Alaoui, "Limit cycle and numerical similations for small and large delays in a predatorprey model with modified Leslie-Gower and Holling-type II schemes," Nonlinear Analysis: Real World Applications, vol. 9, no. 5, pp. 2055-2067, 2008.

[14] Y. X. Xu and M. Y. Huang, "Homoclinic orbits and Hopf bifurcations in delay differential systems with T-B singularity," Journal of Differential Equations, vol. 244, no. 3, pp. 582-598, 2008.

[15] Y. A. Kuznetsov, Elements of Applied Bifurcation Theory, vol. 112 of Applied Mathematical Sciences, Springer, New York, NY, USA, 1995.

[16] F. Y. Lian and Y. T. Xu, "Hopf bifurcation analysis of a predatorprey system with Holling type IV functional response and time delay," Applied Mathematics and Computation, vol. 215, no. 4, pp. 1484-1495, 2009.

[17] J. K. Hale, Theory of Functional Differential Equations, Springer, New York, NY, USA, 1997. 


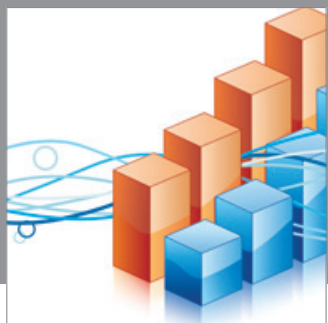

Advances in

Operations Research

mansans

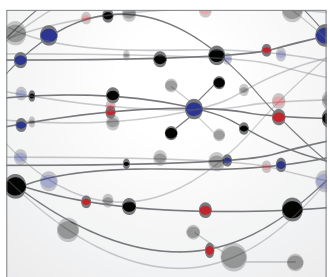

The Scientific World Journal
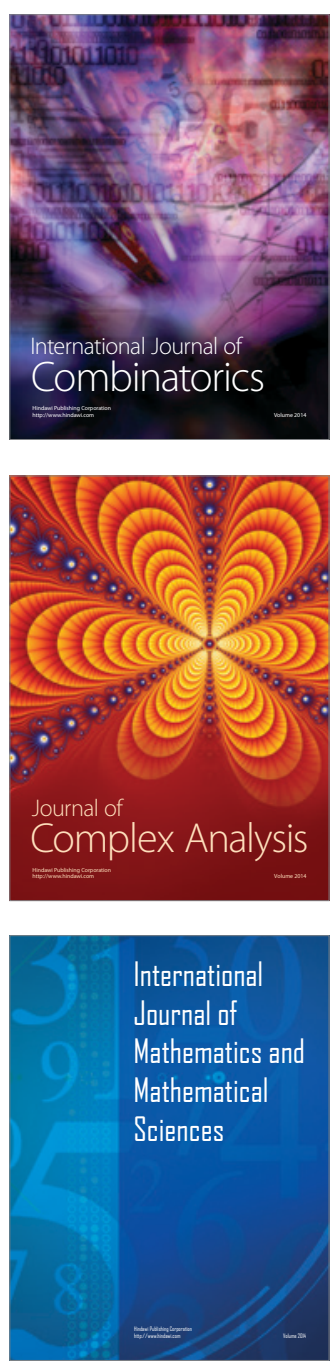
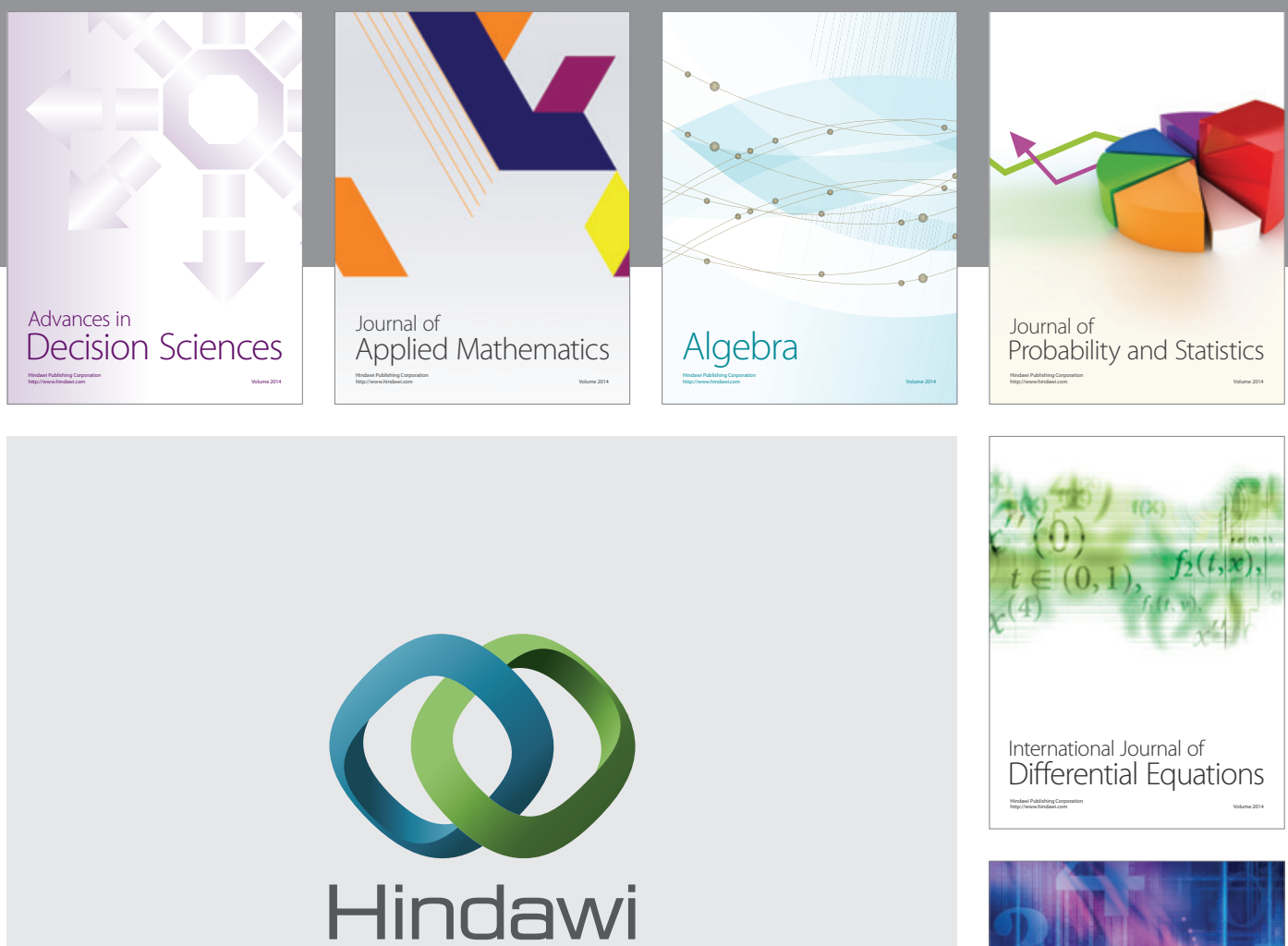

Submit your manuscripts at http://www.hindawi.com
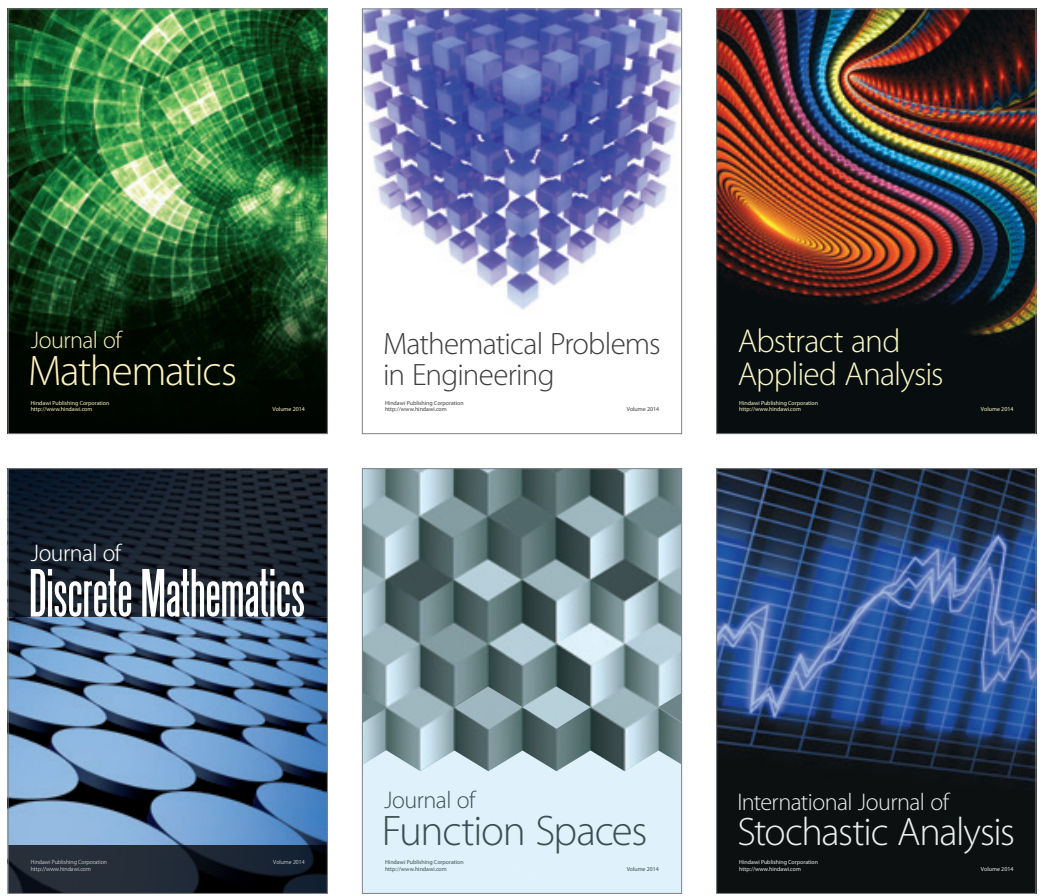

Journal of

Function Spaces

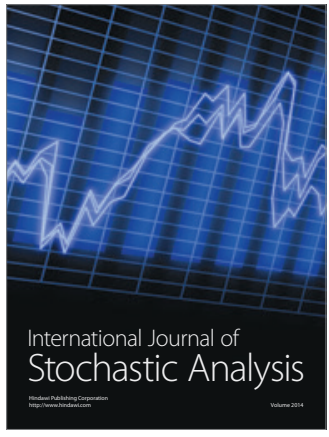

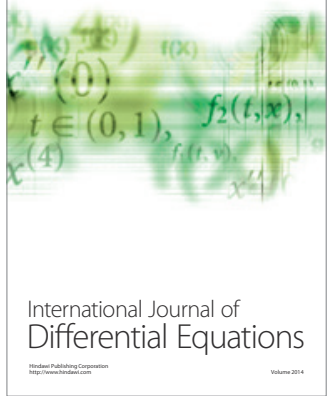
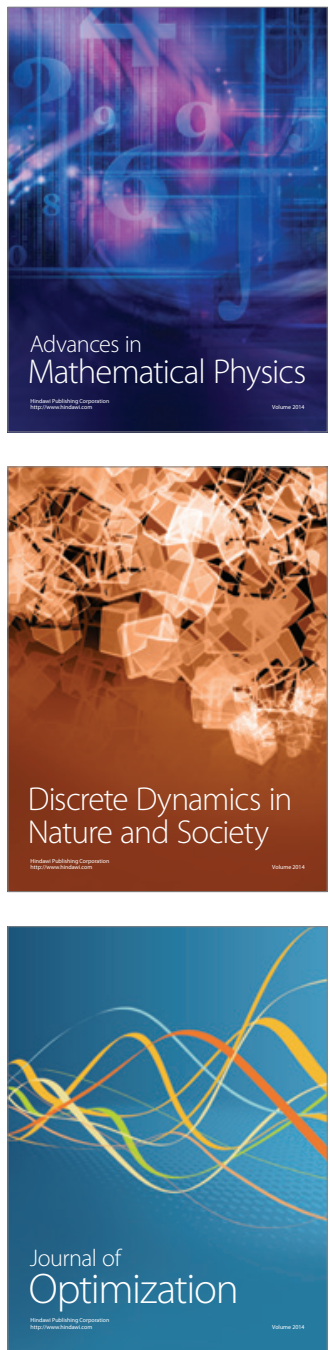